\title{
Intestinal ischemic preconditioning reduces liver ischemia reperfusion injury in rats
}

\author{
TONG-MIN XUE* , LI-DE TAO*, JIE ZHANG, PEI-JIAN ZHANG, XIA LIU, GUO-FENG CHEN and YI-JIA ZHU
}

Institute of General Surgical Research, Second Affiliated Hospital, Yangzhou University, Yangzhou, Jiangsu 225001, P.R. China

Received February 7, 2015; Accepted December 15, 2015

DOI: $10.3892 / \mathrm{mmr} .2016 .4817$

\begin{abstract}
The aim of the current study was to investigate whether intestinal ischemic preconditioning (IP) reduces damage to the liver during hepatic ischemia reperfusion (IR). Sprague Dawley rats were used to model liver IR injury, and were divided into the sham operation group (SO), IR group and IP group. The results indicated that IR significantly increased Bax, caspase 3 and NF- $\mathrm{Bp} 65$ expression levels, with reduced expression of Bcl-2 compared with the IP group. Compared with the IR group, the levels of AST, ALT, MPO, MDA, TNF- $\alpha$ and IL-1 were significantly reduced in the IP group. Immunohistochemistry for Bcl-2 and Bax indicated that $\mathrm{Bcl}-2$ expression in the IP group was significantly increased compared with the IR group. In addition, IP reduced Bax expression compared with the IR group. The average liver injury was worsened in the IR group and improved in the IP group, as indicated by the morphological evaluation of liver tissues. The present study suggested that IP may alleviates apoptosis, reduce the release of pro-inflammatory cytokines, ameloriate reductions in liver function and reduce liver tissue injury. To conclude, IP provided protection against hepatic IR injury.
\end{abstract}

\section{Introduction}

Hepatocellularcarcinomais aleading cause of cancer-associated mortality worldwide (1), with the morbidity and mortality of liver cancer increasing (2). In clinical surgery, liver resection or liver transplantation remains the predominant method of treating liver tumors. The selection of an appropriate method by which to occlude the hepatic vasculature is an important means by which to reduce intraoperative bleeding and improve

Correspondence to: Professor Pejian Zhang, Institute of General Surgical Research, Second Affiliated Hospital, Yangzhou University, 368 Hanjiang Road, Yangzhou, Jiangsu 225001, P.R. China

E-mail: yzu.edu.pjz@163.com

*Contributed equally

Key words: intestinal ischemic preconditioning, ischemia-reperfusion, liver damage surgical safety. The methods by which the hepatic artery pedicle may be blocked include hepatic vascular occlusion, semi-hepatic vascular occlusion and hepatic blood flow occlusion. Ischemia-reperfusion (IR) is an unavoidable consequence of certain surgical procedures such as partial liver resection and organ transplantation (3), and frequently results in varying degrees of hepatic ischemia reperfusion injury (HIRI). Liver IR injury is a phenomenon in which cellular damage due to hypoxia is exacerbated following the return of blood flow and the restoration of oxygen delivery. This phenomena remains an important clinical problem during shock, hepatic resection and liver transplantation (4). Reperfusion is required to avoid irreversible damage, however, it may produce oxygen free radicals via the hypoxanthine-xanthine oxidase system, alter the distribution of ions, edema and cellular acidosis, and culminate in the loss of circulation and increasing the injury (5). IR injury is a complicated process involving various associated mechanisms including apoptosis and pro-inflammatory cytokines. Apoptosis, as one of the most important mechanisms associated with IR injury, serves a vital role in the initiation and progression of IR injury (6).

In order to protect the ischemic areas resulting from IR, various methods have been utilized, including ischemic preconditioning (IP). IP refers to the induction of one or more transient IR episodes, resulting in the induction of endogenous protective mechanisms and conferring significant tolerance to longer duration ischemic injury. Przyklenk first described remote ischemic preconditioning (RIPC) in 1993, demonstrating that brief occlusion of the circumflex artery protects the myocardium from subsequent continuous IR injury (7). Subsequently, this method has been demonstrated to be an effective way to protect the liver without direct stress (4). RIPC involves brief periods of ischemia followed by reperfusion in a single organ or tissue, which subsequently provides protection to a remote organ or tissue suffering from a prolonged ischemic injury (8). Increasing evidence suggests that intestinal IP is able to reduce remote organ injuries, and remote IP is easily applied and safe in the clinical setting. In addition, remote IP is able to attenuate systemic inflammatory response syndrome, and increase systemic tolerance to IR, providing cytoprotection in critical organs, including the liver (9). Although remote intestinal IP has been indicated to be beneficial in liver IR, the exact mechanism remains to be fully elucidated. The current study hypothesized that remote intestinal IP may be a prophylactic factor in the prevention of distant liver injury induced by 
IR. Therefore, the aim of the current study was to elucidate the molecules and potential mechanisms involved in the protective effects of intestinal IP in reducing liver injury.

\section{Materials and methods}

Sprague Dawley (SD) rats and ethics statement. A total of 15 male SD rats (weight, 250-300 g, age, 10-12 weeks) were obtained from the Experimental Animal Center of Yangzhou College (Yangzhou, China). The study was approved by the Animal Care and Ethics Committee of The Second Affiliated Hospital, Yangzhou College (Yangzhou, China). All rats were given free access to food and water, maintained at $20^{\circ} \mathrm{C}$ and $50 \%$ humidity in a $12 \mathrm{~h}$-light/12 h-dark cycle.

Rat model of ischemia-reperfusion. The rats were divided into the sham operation group (SO group), ischemia-reperfusion group (IR group) and the remote intestinal IP+IR group (IP group), with 5 rats in each group. All animals were fasted for $12 \mathrm{~h}$ prior to surgery (free access to water), and anesthetized using an intraperitoneal injection of pentobarbital anesthesia $(35 \mathrm{mg} / \mathrm{kg}, 2 \%)$.

A $3 \mathrm{~cm}$ incision was made in the abdomen, and the superior mesenteric artery (SMA) and the hepatic pedicle were isolated. In the $\mathrm{SO}$ group, rats were anesthetized, an abdominal incision was made and the SMA and the hepatic pedicle were isolated and then the incision closed, with the incision open for the same duration as the IR and IP groups. In the IR group, the incision was made and the liver pedicle clamped to block $70 \%$ of blood flow to the liver for $30 \mathrm{~min}$, followed by $3 \mathrm{~h}$ reperfusion and the closing of the incision. In the IP group, the SMA was clamped in two cycles of $10 \mathrm{~min}$ ischemia and 10 min reperfusion, followed by the clamping of the liver pedicle for $30 \mathrm{~min}$ and $3 \mathrm{~h}$ of reperfusion, and the closing of the incision. The rats were allowed to recover and wake from surgery and $3 \mathrm{~h}$ after reperfusion, the rats were anaesthetized by intraperitoneal injection of pentobarbital (Sigma-Aldrich) and sacrificed by cervical dislocation. The liver tissues and blood were used for the following experiments.

Serum aspartate aminotransferase (AST) and alanine transaminase (ALT) testing. Total blood was collected and centrifuged at $1,788 \mathrm{x} \mathrm{g}$ for $5 \mathrm{~min}$. The serum was extracted $3 \mathrm{~h}$ following reperfusion and used for the measurement of AST and ALT with a standard automatic biochemistry analyzer model (BS-800; Mindray Medical International Ltd., Shenzhen, China).

Measurement of myeloperoxidase (MPO) and malondialdehyde (MDA) activity. The reperfused liver tissues and serum were frozen immediately $3 \mathrm{~h}$ after reperfusion and stored at $-80^{\circ} \mathrm{C}$ until assessment. MPO and MDA activities were measured using an MPO Colorimetric Activity Assay kit and a Micro-MDA Assay Reagent kit (Kaiji Biological Technology Development Co., Ltd., Nanjing, China). The liver tissues were homogenized in $50 \mathrm{mmol} / \mathrm{l}$ potassium phosphate buffer, $\mathrm{pH}$ 6 , containing $0.5 \%$ hexadecyltrimethyl ammonium bromide. The homogenates were centrifuged for $10 \mathrm{~min}$ at $12,500 \mathrm{x} \mathrm{g}$ at $4^{\circ} \mathrm{C}$. The supernatants were collected and reacted with $0.167 \mathrm{~g} / \mathrm{l}$ o-dianisodine dihydrochloride and $0.0005 \% \mathrm{H}_{2} \mathrm{O}_{2}$ in
$50 \mathrm{mmol} / \mathrm{l}$ phosphate buffer, and the absorbance was determined spectrophotometrically (UV-2450; Shimadzu Co., Ltd., Kyoto, Japan) at $460 \mathrm{~nm}$.

Determination of tissue necrosis factor- $\alpha(T N F-\alpha)$ and interleukin-1 $\beta(I L-1 \beta)$. The serum was frozen immediately $3 \mathrm{~h}$ after reperfusion and stored at $-80^{\circ} \mathrm{C}$ until assessment. TNF- $\alpha$ and IL- $1 \beta$ levels were determined using ELISA kits (R\&D Systems, Inc., Minneapolis, MN, USA) according to the manufacturer's instructions. An ELISA microplate reader (Hamilton Bonaduz AG, Bonaduz, Switzerland) was used. The results were expressed as $\mathrm{pg} / \mathrm{ml}$.

Western blot analysis. The rat liver tissue $(100 \mathrm{mg})$ was homogenized in liquid nitrogen, and lysis buffer [Tris ( $\mathrm{pH} 8.1)$, $1 \%$ SDS, sodium pyrophosphate, $\beta$-glycerophosphate, sodium orthovanadate, sodium fluoride, EDTA, leupeptin;Santa Cruz Biotechnology, Inc., Dallas, TX, USA].containing phosphatase inhibitors (Nanjing Kangji Biological Technology Development Co., Ltd.) were added. The protein concentration of the samples was determined using a Bicinchoninic Acid Protein Assay kit (Santa Cruz Biotechnology, Inc.). The protein extracts $(30 \mu \mathrm{g})$ were electrophoresed on $10 \%$ sodium dodecyl sulfate-polyacrylamide gel (Nanjing Kangji Biological Technology Development Co., Ltd.), transferred onto polyvinylidene fluoride membranes (Bio-Rad Laboratories, Inc., Hercules, CA, USA) and incubated for $1 \mathrm{~h}$ in Tris-buffered saline (TBS) containing 5\% nonfat milk and $0.1 \%$ Tween-20. Subsequently, the membranes were incubated overnight at $4^{\circ} \mathrm{C}$ with the following primary antibodies: Monoclonal anti-nuclear factor- $\kappa \mathrm{B}(\mathrm{NF}-\kappa \mathrm{B}) \mathrm{p} 65$ (1:500 dilution; cat. no. 558393; BD Biosciences), monoclonal anti-Bax (1:1,000 dilution; cat. no. 610983; BD Biosciences, Franklin Lakes, NJ, USA), monoclonal anti-Bcl-2 (1:1,000 dilution; cat. no. 610538; BD Biosciences), monoclonal anti-Capase-3, (1:1,000; cat. no. 610322; BD Biosciences). Following washing in TBS with $0.1 \%$ Tween-20, the membranes were incubated for $1 \mathrm{~h}$ at room temperature with horseradish peroxidase-conjugated goat anti-rabbit IgG antibody (1:1,000; cat. no. sc-2004; Santa Cruz Biotechnology, Inc.). Immunoreactivity was detected using an enhanced chemiluminescence kit (Santa Cruz Biotechnology Inc.) and visualized by autoradiography. The level of $\beta$-actin (1:1,000; cat. no. sc-1616; Santa Cruz Biotechnology Inc.) was used as a loading control and the optical density of each band was measured using ImageJ (National Institutes of Health, Bethesda, MD, USA).

Immunohistochemistry for Bcl-2 and Bax. Immunohistochemistry was performed on $10 \mu \mathrm{m}$-thick sections The liver tissue was dehydrated with a graded series of alcohol. Subsequently, paraffin was used for embedding tissue. Following incubation in $3 \% \mathrm{H}_{2} \mathrm{O}_{2}(5-10 \mathrm{~min})$, sections were blocked using 10\% normal goat serum (Santa Cruz Biotechnology, Inc.) in phosphate-buffered saline-Tween-20 $(0.2 \%)$ for $10 \mathrm{~min}$. Slides were incubated with mouse anti-Bcl-2 and Bax (BD Biosciences, Franklin Lakes, NJ, USA cat. no. $610204 ; 1: 1,000$, diluted in $1 \% \mathrm{BSA} / \mathrm{PBS}$ ) at $4^{\circ} \mathrm{C}$ overnight followed by incubation with horseradish peroxidase-conjugated rabbit anti-mouse Ig and goat anti-rabbit Ig (Dako, Glostrup, Denmark), 1:100 diluted in 1\% BSA/1\% 

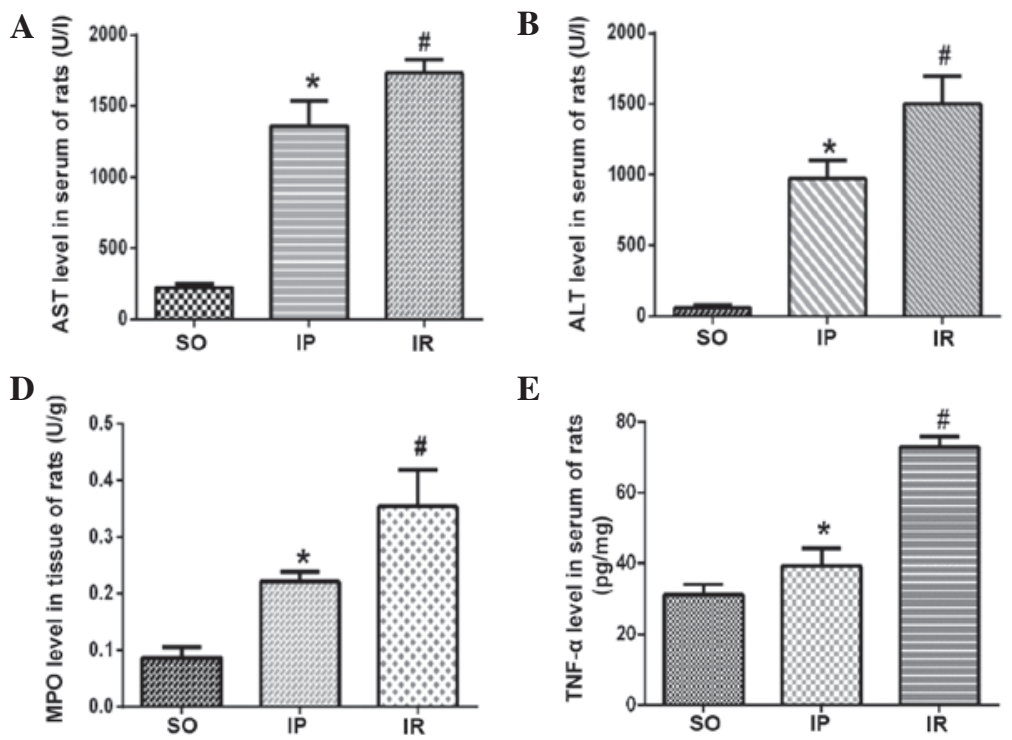

$\mathbf{E}$

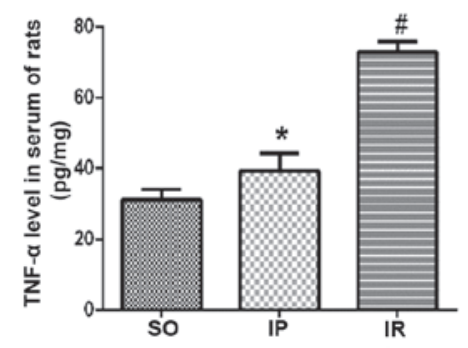

C

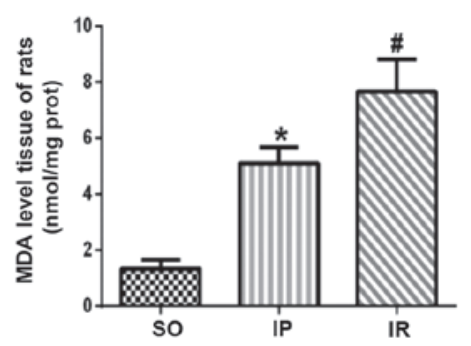

$\mathbf{F}$

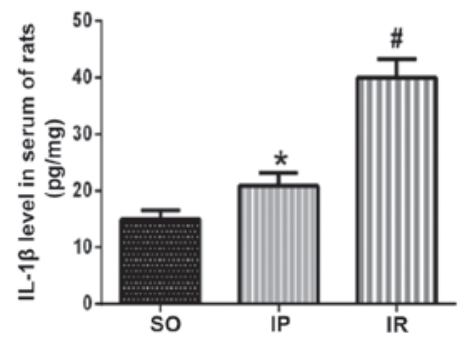

Figure 1. Expression levels of (A) AST, (B) ALT, (C) MDA, (D) MPO, (E) TNF- $\alpha$ and (F) IL-1 $\beta$. Data are presented as the mean \pm standard deviation (n=5). ${ }^{*} \mathrm{P}<0.05$ vs. the SO group, ${ }^{\#} \mathrm{P}<0.05$ vs. the SO group. AST, aspartate aminotransferase; ALT, alanine transaminase; MDA, malondialdehyde; MPO, myeloperoxidase; TNF- $\alpha$, tissue necrosis factor- $\alpha$; IL-1 $\beta$, interleukin-1 $\beta$; SO, sham group; IP, ischemic preconditioning group; IR, ischemia reperfusion group.

albumin/PBS. Diaminobenzidine was used to develop the staining reaction and nuclear counterstaining was performed with haematoxylin. As a negative control, sections were incubated without the primary antibody. Positive cytoplasmic yellow-brown staining was imaged using a light microscope (Olympus BX-51; Olympus Corporation, Tokyo, Japan). Bcl-2 and Bax-positive cells were counted at magnification, $\mathrm{x} 400$, in five sections from each animal. The integrated optical density (IOD) was calculated from the images.

Histology. Standard hematoxylin and eosin staining was performed on representative $3 \mathrm{~mm}$ sections from each rat, for general histopathologic evaluation of each group according to Suzuki's classification (10), in which sinusoidal congestion, hepatocyte necrosis and ballooning degeneration are graded from 0 to 4 . Following this, the total liver injury scores were calculated by totaling the individual scores for each category.

Statistical analysis. All data are expressed as the mean \pm standard deviation, and were analyzed by one way analysis of variance. Data analysis was performed using SPSS software, version 18.0 (SPSS, Inc., Chicago, IL, USA). P<0.05 was considered to indicate a statistically significant difference. All experiments were performed three times.

\section{Results}

AST, ALT, MDA, MPO, TNF- $\alpha$, and IL-1 $\beta$ levels in rats. As presented in Fig. 1A and B, the serum AST and ALT levels in the IR group were significantly increased compared with the SO group $(\mathrm{P}<0.05)$. IP decreased the serum AST levels compared with the IR group. As shown in Fig. 1C, the MDA levels in the IR group were significantly increased compared with the SO group. IP resulted in a reduced MDA level compared with the IR group. The MPO levels in the IR group were significantly increased compared with the SO group, and in the IP group, MPO levels were reduced compared with the
IR group (Fig. 1D). As presented in Fig. 1E and F, the serum TNF- $\alpha$ and IL-1 $\beta$ levels in the IR group were significantly increased compared with the SO group, and IP reduced these levels compared with the IR group $(\mathrm{P}<0.05)$.

Western blotting of Bcl-2, Bax, caspase 3 and NF- $\kappa B p 65$. Fig. 2 presents the levels of Bcl-2, Bax, caspase 3 and NF- $\mathrm{Bp} 65$. Compared with the SO group, Bcl-2 levels were increased in the IR group, however, they were reduced compared with the IP group $(\mathrm{P}<0.05)$. IP pretreatment resulted in elevated $\mathrm{Bcl}-2$ expression and attenuated Bax, caspase 3 and NF- $\kappa \mathrm{Bp} 65$ expression levels compared with the IR group ( $\mathrm{P}<0.05$; Fig. 2).

Immunohistochemistry for Bcl-2 and Bax. The expression of Bcl-2 and Bax in liver tissue was examined, with the IOD calculated for each group. As presented in Fig. 3, the Bcl-2 expression in the IR group was significantly increased compared with the SO group $(\mathrm{P}<0.05)$. Furthermore, IP markedly increased Bcl-2 expression compared with the IR group $(\mathrm{P}<0.01)$. As presented in Fig. 4, Bax expression in the IR group was significantly greater than that in the SO group $(\mathrm{P}<0.05)$, and IP significantly reduced Bax expression compared with the IR group $(\mathrm{P}<0.05)$.

Liver histology. The alterations in liver tissue structure were observed by light microscopy. As presented in Fig. 5A, in the SO group, hepatocytes and sinusoidal endothelial cells exhibited no apparent degeneration or necrosis, and the central venous and periportal structures are well defined. In the IR group, swollen liver cells, alterations in the morphology of cell nuclei, congestion of hepatic sinusoids, inflammatory cell infiltration and visible liver structural damage were observed. The observed liver injury was improved in the IP group, compared with the IR group, as indicated by the representative images of the liver tissues. As shown in Fig. 5B, the liver injury scores in the IR group were significantly increased compared with the SO group $(\mathrm{P}<0.05)$, and 

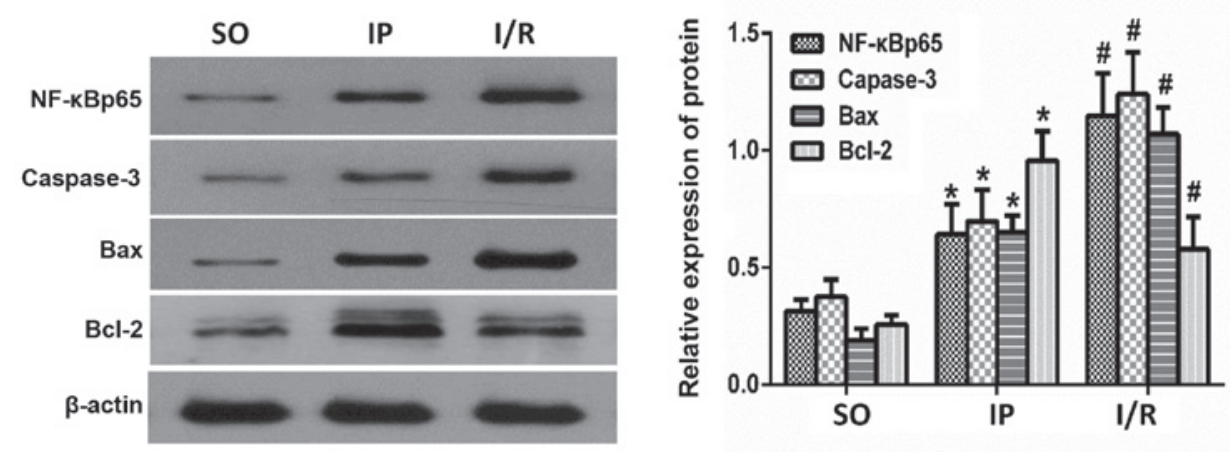

Figure 2. Protein expression of NF- $\kappa$ Bp65, caspase 3, Bax and Bcl-2 measured in the liver tissue of rats. Data are presented as the mean \pm standard deviation

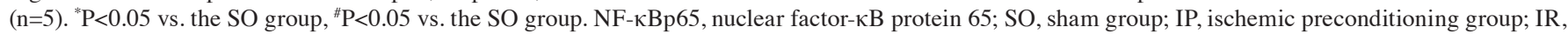
ischemia reperfusion group.
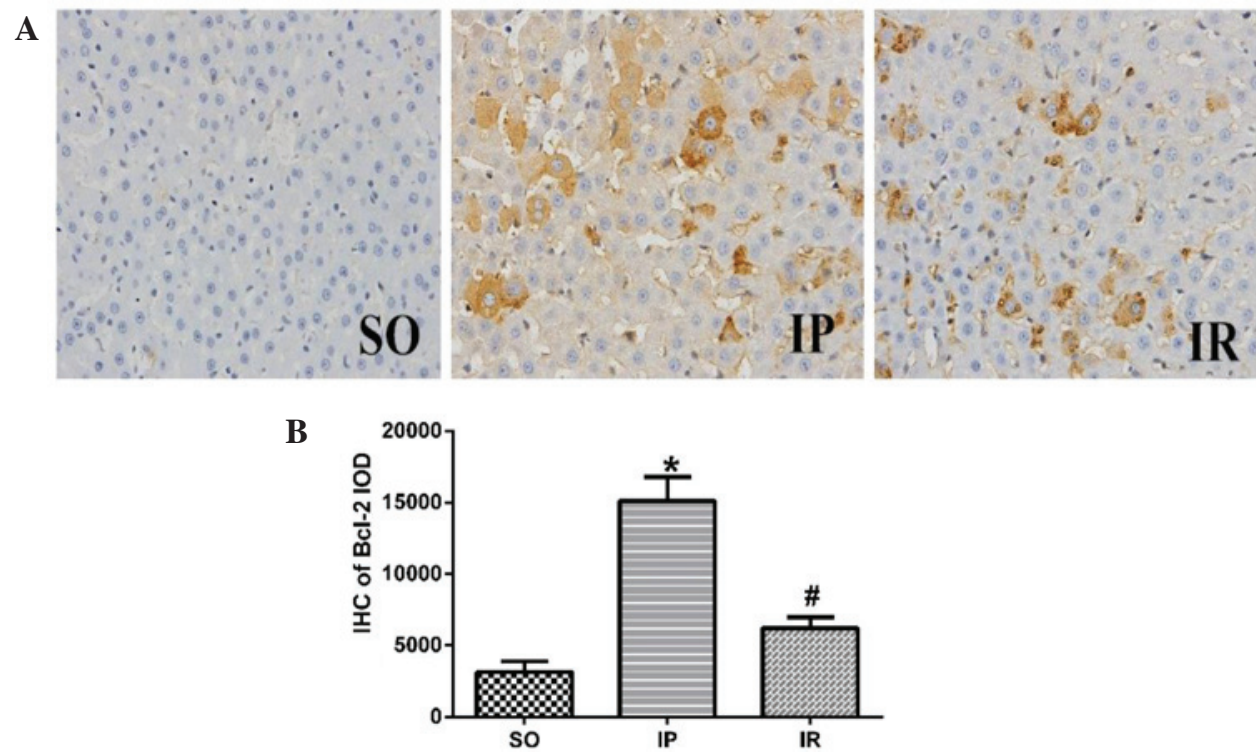

Figure 3. Bcl-2 expression in the liver tissue of rats (magnification, $\mathrm{x} 400$ ). (A) Representative images of $\mathrm{Bcl}-2$ expression in the liver tissue of rats. (B) IOD of $\mathrm{Bcl}-2$ expression in the liver tissue of rats. Data are presented as the mean \pm standard deviation $(\mathrm{n}=5)$. ${ }^{\mathrm{P}} \mathrm{P}<0.05$ vs. the SO group, ${ }^{~} \mathrm{P}<0.05$ vs. the $\mathrm{SO}$ group. SO, sham group; IP, ischemic preconditioning group; IR, ischemia reperfusion group; IHC, immunohistochemistry; IOD, integrated optical density.

were reduced in the IP group compared with the IR group $(\mathrm{P}<0.05)$.

\section{Discussion}

In clinical surgery, liver resection or liver transplantation remains the predominant method for treating liver tumors (11). The selection of an appropriate method to occlude the hepatic vasculature is an important factor in reducing intraoperative bleeding and improve surgical safety. IR is unavoidable in certain surgical procedures such as partial liver resections and organ transplantation. The anoxic injury of oxygen-dependent cells is the predominant injury process in the ischemic phase. Once the blood flow and oxygen supply are re-established, reperfusion enhances the injury resulting from the ischemic period, aggravating the damage at the cellular level, with an injurious inflammatory response involved (12-14). The severity of the cellular injury may lead to either hepatocyte necrosis or apoptosis (15).
IP is a method of repeated transient intestinal ischemia reperfusion, which induces the liver to produce endogenous protective mechanisms by which it is able to tolerate longer ischemic injuries. The mechanism by which IP reduces liver IR injury remains to be fully elucidated. Further studies regarding the mechanisms associated with IP-mediated protection may promote its use in liver resection and liver transplantation.

The levels of apoptosis in hepatocytes reflects alterations in liver function and reserve capacity, and is an important pathological feature of HIRI, associated with postoperative residual liver dysfunction (16). Numerous factors are able to trigger apoptosis, with the process controlled by a number of regulatory molecules which are mediated by apoptotic signaling $(17,18)$. In the current study, in addition to enhancing the defense capacity of liver cells against oxidative stress induced by IR injury, IP treatment was observed to upregulate the anti-apoptotic protein $\mathrm{Bcl}-2$ and reduce the expression levels of pro-apoptotic proteins, such as Bax and caspase 3 . The Bcl-2 family of proteins are closely associated with 
A

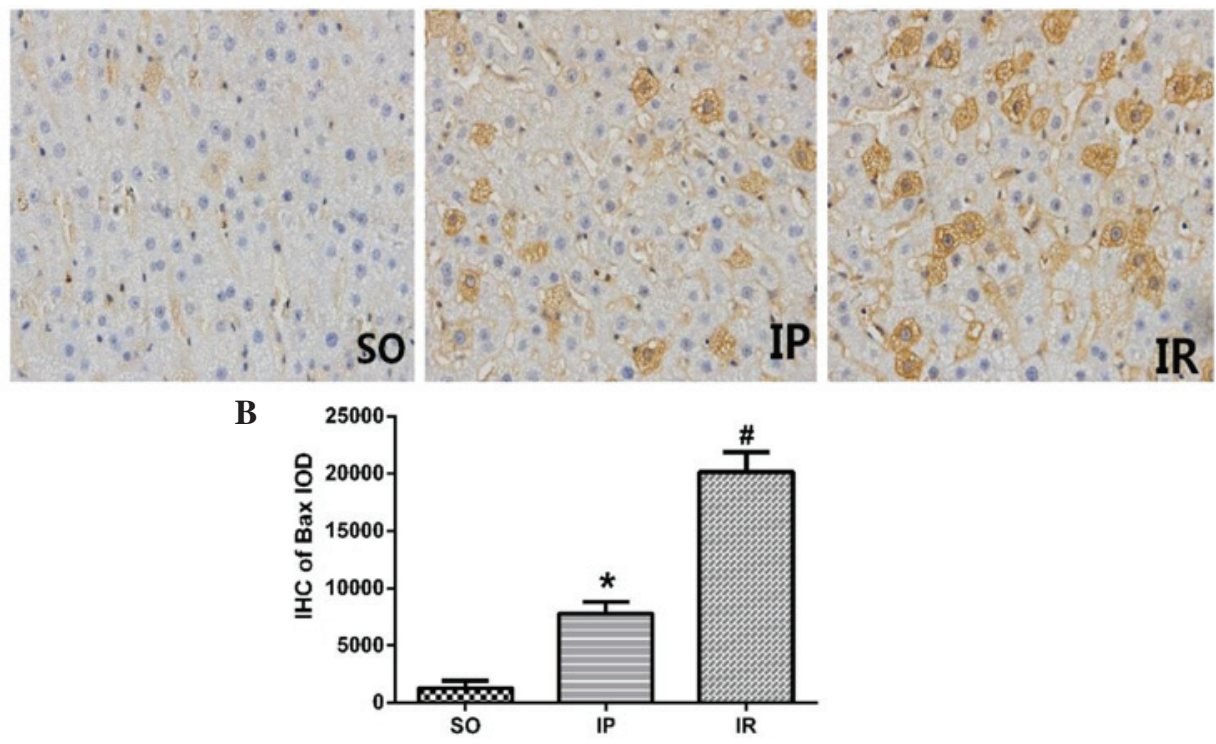

Figure 4. Bax expression in the liver tissue of rats (magnification, $x 400$ ). (A) Representative images of Bax expression in the liver tissue of rats. (B) IOD of Bax expression in the liver tissue of rats. Data are presented as the mean \pm standard deviation $(\mathrm{n}=5)$. ${ }^{*} \mathrm{P}<0.05$ vs. the $\mathrm{SO}$ group, ${ }^{*} \mathrm{P}<0.05$ vs. the $\mathrm{SO}$ group. $\mathrm{SO}$, sham group; IP, ischemic preconditioning group; IR, ischemia reperfusion group; IHC, immunohistochemistry; IOD, integrated optical density.

A
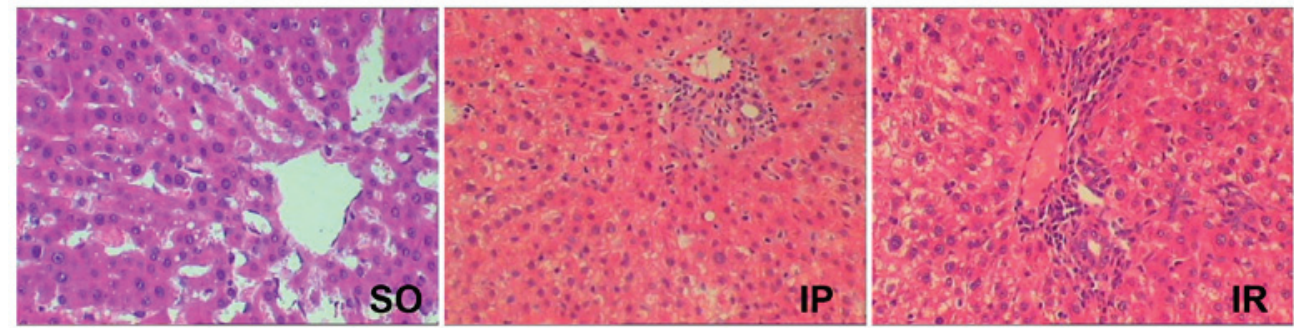

B

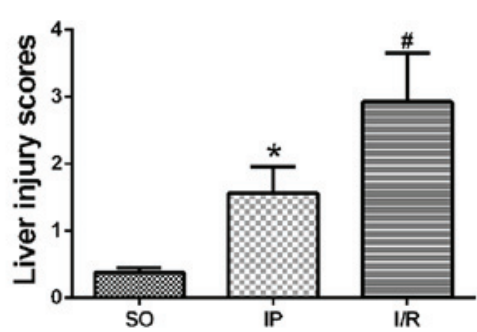

Figure 5. Hemetoxylin and eosin staining of the alterations in the liver tissue of rats. (A) Representative images of the morphological alterations in the liver tissue of rats (magnification, $\mathrm{x} 400$ ). (B) Liver injury scores in rats. Data are presented as the mean \pm standard deviation $\left(\mathrm{n}=5\right.$ ). ${ }^{*} \mathrm{P}<0.05 \mathrm{vs}$. the $\mathrm{SO}$ group, ${ }^{\#} \mathrm{P}<0.05$ vs. the SO group. SO, sham group; IP, ischemic preconditioning group; IR, ischemia reperfusion group.

apoptosis $(19,20)$. The family is divided into three subfamilies, including the anti-apoptotic members Bcl-2, Bcl-XL and Mcl-1, the pro-apoptotic members Bax and Bak, and the only pro-apoptotic BH3 domain subfamily members (21). The Bcl-2 family of proteins, including Bcl-2 (anti-apoptotic) and Bax (pro-apoptotic), mediate apoptosis by opening or closing the mitochondrial permeability transition pore. Downregulation of the ratio of Bcl-2 to Bax has been reported to be correlated with the activation of the caspase cascade, which further cleaves or activates downstream regulators, such as caspase 3, resulting in the subsequent apoptotic events (22). The anti-apoptotic mechanisms associated with $\mathrm{Bcl}-2$ are as follows: i) As an antioxidant, $\mathrm{Bcl}-2$ regulates the cell redox status, blocking oxidative damage to cell components; ii) influences cell membrane transport, altering the distribution of $\mathrm{Ca}^{2+}$; iii) inhibits the release of cytochrome $\mathrm{C}$ from mitochondria into the cytoplasm; and iv) protects the cell nucleus from damage and inhibits DNA fragmentation. Bax is the most widely studied of the pro-apoptotic proteins (23), and the mechanisms associated include: i) Bax forms heterologous dimers with Bcl-2 to antagonize its anti-apoptotic effects, to promote $\mathrm{Ca}^{2+}$ release from the endoplasmic reticulum; and ii) interacts with cytochrome $\mathrm{C}$ and activates caspase signaling transduction pathways, which indirectly promote apoptosis (24). Bax may be activated in HIRI, with the activation of Bax resulting in the exposure of a carboxy-terminal domain, enabling binding to the outer mitochondrial membrane. This leads to the release of caspase 3 and additional pro-apoptotic 
factors from mitochondria, such as apoptosis inducing factor, thus contributing to the formation of apoptotic bodies and inducing apoptosis (25). The results of the present study indicated that Bax expression was downregulated, whereas Bcl-2 expression was upregulated in the IP group compared with the IR group, suggesting a reduction in apoptosis in the liver tissue. These results suggest that intestinal IP may suppress IR-induced apoptosis by the upregulation of the anti-apoptotic protein Bcl-2 and the downregulation of the pro-apoptotic protein Bax. In addition, the immunohistochemical staining indicated a significant difference in the expression levels of Bcl-2 and Bax in each group. Compared with the IR group, the IP group exhibited reduced apoptosis, suggesting that through the induction of Bcl-2 expression in the liver cells, IP inhibits Bax activation, and thereby reduces the apoptosis of liver cells. In the apoptotic process, signals from the death ligand are received on the cell surface and activate intracellular proteins such as caspases, leading to the degradation of specific target proteins and resulting in cell death (26). Numerous studies have demonstrated that ischemia induces cell degradation and apoptosis, accompanied by reduced levels of Bcl-2 and increased levels of cleaved caspase 3 in the ischemic core (27-29). In the current study, the expression of cleaved caspase 3 in liver tissue was detected. The results demonstrated that intestinal IP attenuated the increase in caspase 3 protein expression compared with the IR group. As an executioner of apoptosis, caspase 3 serves a pivotal role in apoptosis, thus the protective role of intestinal IP is associated with the inhibition of caspase 3. Intestinal IP may suppress IR-associated apoptosis via the upregulation of $\mathrm{Bcl}-2$ and the downregulation of caspase 3 and Bax.

$\mathrm{NF}-\kappa \mathrm{B}$ is a nuclear transcription factor that regulates the expression of numerous genes that are critical for the regulation of apoptosis, viral replication, tumorigenesis, inflammation and various autoimmune diseases. $N F-\kappa B$ is normally sequestered in the cytoplasm by a family of inhibitory proteins known as inhibitory- $\kappa$ Bs $(\mathrm{I} \kappa \mathrm{Bs})$. A wide variety of stimuli, which have been extensively studied over the past two decades (30), result in $\mathrm{I} \kappa \mathrm{B} \alpha$ phosphorylation, a process that is followed by its ubiquitination and subsequent degradation. The loss of $\mathrm{I} \kappa \mathrm{B} \alpha$ results in the release of the free $\mathrm{NF}-\kappa \mathrm{B}$ subunit, p65, which translocates from the cytoplasm to the nucleus, where it triggers the transcription of multiple inflammatory genes, including cytokines (TNF- $\alpha$, IL-1 $\beta$, iNOS and COX2), chemokines and adhesion molecules $(31,32)$. In the current study, the levels of TNF- $\alpha$ and IL-1 $\beta$ were observed to be significantly increased in the IR group. However, in the rats that received IP, the levels of TNF- $\alpha$ and IL-1 $\beta$ were significantly reduced. These observations indicate that IP may inhibit $\mathrm{NF}-\kappa \mathrm{Bp} 65$ activation, mitigate liver injury and the infiltration of inflammatory cells.

MDA is the final product of lipid peroxidation and an important parameter in the evaluation of IR-associated oxidative injury. Lipid peroxidation leads to impaired cell membrane permeability, reduced membrane potential and cellular damage. Cell damage further intensifies with MDA formation $(33,34)$. In the current study, IP was observed to significantly reduce the IR-associated increase in MDA levels in liver tissue, compared with the control group. MPO is another known oxidant within cells. In the presence of chloride ions, MPO reduces hydrogen peroxide to hypochlorous acid. Hypochlorous acid is a powerful oxidant and leads to tissue damage as it is highly reactive with several biological molecules (35). The current study indicated that IP significantly reduced the IR-associated increase in MPO levels in liver tissue, compared with the IR group. In addition, IP significantly reduced the levels of MDA and MPO expression following IR, indicating that IP is effective in the reduction of liver IR injury.

Previous studies have indicated that IP results in significant improvements in liver function, as indicated by serum AST and ALT levels $(36,37)$. The current study indicated that AST and ALT levels were increased following reperfusion, indicating that liver injury occurs during reperfusion. In the groups in which protection was promoted by IP, the AST and ALT levels were reduced compared with the IR group, indicating that IP reduces liver IR injury. In addition, liver histology was investigated for liver tissue alterations and liver injury scores. In the present study, the average liver tissue alterations and the liver injury scores were worsened in the IR group and improved in the IP group. Therefore, it may be proposed that intestinal IP inhibits IR-mediated liver injury, as indicated by histologic and cytologic features.

In conclusion, IP provided protection against hepatic IR injury by upregulating the anti-apoptotic protein Bcl-2 and reducing the expression of the pro-apoptotic proteins, Bax and caspase 3 . In addition, IP reduces NF- $\kappa$ Bp65, inhibits the release of pro-inflammatory cytokines and ameliorates the destructive capacity of oxygen free radicals by reducing MDA and MPO expression and reducing AST and ALT.

\section{Acknowledgements}

The current study was supported in part by grants from the Yangzhou Science and Technology Program (grant no. YZ2014064) and the Project Sponsored by the Institute of General Surgical Research, Second Affiliated Hospital, Yangzhou University.

\section{References}

1. Chen JG and Zhang SW: Liver cancer epidemic in China: Past, present and future. Semin Cancer Biol 21: 59-69, 2011.

2. Taura N, Ichikawa T, Miyaaki H, Ozawa E, Tsutsumi T, Tsuruta S, Kato Y, Goto T, Kinoshita N, Fukushima M, et al: Frequency of elevated biomarkers in patients with cryptogenic hepatocellular carcinoma. Med Sci Monit 19: 742-750, 2013.

3. Castro e Silva O, Sankarankutty AK, Martinelli AL, Souza FF, Teixeira AC, Feres O, Mente ED, Oliveira GR, Akita R, Muglia V, et al: Therapeutic effect of hyperbaric oxygen in hepatic artery thrombosis and functional cholestasis after orthotopic liver transplantation. Transplant Proc 38: 1913-1917, 2006.

4. Wang Y, Shen J, Xiong X, Xu Y, Zhang H, Huang C, Tian Y, Jiao $\mathrm{C}$, Wang $\mathrm{X}$ and Li $\mathrm{X}$ : Remote ischemic preconditioning protects against liver ischemia-reperfusion injury via heme oxygenase-1-induced autophagy. PLoS One 9: e98834, 2014.

5. Flynn EP and Auer RN: Eubaric hyperoxemia and experimental cerebral infarction. Ann Neurol 52: 566-572, 2002.

6. Lei B, Popp S, Capuano-Waters C, Cottrell JE and Kass IS: Lidocaine attenuates apoptosis in the ischemic penumbra and reduces infarct size after transient focal cerebral ischemia in rats. Neuroscience 125: 691-701, 2004.

7. Przyklenk K, Bauer B, Ovize M, Kloner RA and Whittaker P: Regional ischemic 'preconditioning' protects remote virgin myocardium from subsequent sustained coronary occlusion. Circulation 87: 893-899, 1993. 
8. Abu-Amara M, Yang SY, Quaglia A, Rowley P, Tapuria N, Seifalian AM, Fuller BJ and Davidson BR: Effect of remote ischemic preconditioning on liver ischemia/reperfusion injury using a new mouse model. Liver Transpl 17: 70-82, 2011

9. Li C, Li YS, Xu M, Wen SH, Yao X, Wu Y, Huang CY, Huang WQ and Liu KX: Limb remote ischemic preconditioning for intestinal and pulmonary protection during elective open infrarenal abdominal aortic aneurysm repair: A randomized controlled trial. Anesthesiology 118: 842-852, 2013.

10. Suzuki S, Toledo-Pereyra LH, Rodriguez FJ and Cejalvo D: Neutrophil infiltration as an important factor in liver ischemia and reperfusion injury. Modulating effects of FK506 and cyclosporine. Transplantation 55: 1265-1272, 1993.

11. Dimick JB, Cowan JA Jr, Knol JA and Upchurch GR Jr: Hepatic resection in the United States: Indications, outcomes, and hospital procedural volumes from a nationally representative database. Arch Surg 138: 185-191, 2003.

12. Tsalkidou EG, Tsaroucha AK, Chatzaki E, Lambropoulou M, Papachristou F, Trypsianis G, Pitiakoudis M, Vaos G and Simopoulos C: The effects of apigenin on the expression of Fas/FasL apoptotic pathway in warm liver ischemia-reperfusion injury in rats. Biomed Res Int 2014: 157216, 2014.

13. Lichtman SN and Lemasters JJ: Role of cytokines and cytokine-producing cells in reperfusion injury to the liver. Semin Liver Dis 19: 171-187, 1999.

14. Maurya DK, Devasagayam TP and Nair CK: Some nove approaches for radioprotection and the beneficial effect of natural products. Indian J Exp Biol 44: 93-114, 2006.

15. Kerr JF, Winterford CM and Harmon BV: Apoptosis. Its significance in cancer and cancer therapy. Cancer 73: 2013-2026, 1994

16. Malhi H, Gores GJ and Lemasters JJ: Apoptosis and necrosis in the liver: A tale of two deaths? Hepatology 43 (Suppl 1): S31-S44, 2006.

17. Hotchkiss RS, Strasser A, McDunn JE and Swanson PE: Cell death. N Engl J Med 361: 1570-1583, 2009.

18. Wang Z, Ji Y, Wang S, Wang R, Li Z, Kang A, Xu H, Shi M and Zhao M: Protective effect of intestinal ischemic preconditioning on ischemia reperfusion-caused lung injury in rats. Inflammation 38: 424-432, 2015.

19. Cleary ML, Smith SD and Sklar J: Cloning and structural analysis of cDNAs for bcl-2 and a hybrid bcl-2/immunoglobulin transcript resulting from the $\mathrm{t}(14 ; 18)$ translocation. Cell 47 19-28, 1986.

20. Chen W, Fu XB, Ge SL, Sun TZ, Zhou G, Han B, Du YR, Li HH and Sheng ZY: Intravenous acid fibroblast growth factor protects intestinal mucosal cells against ischemia-reperfusion injury via regulating Bcl-2/Bax expression. World J Gastroenterol 11 3419-3425, 2005.

21. Packham G and Stevenson FK: Bodyguards and assassins: Bcl-2 family proteins and apoptosis control in chronic lymphocytic leukaemia. Immunology 114: 441-449, 2005.

22. Hardwick JM and Soane L: Multiple functions of BCL-2 family proteins. Cold Spring Harb Perspect Biol 5: a008722, 2013.
23. Oltvai ZN, Milliman CL and Korsmeyer SJ: Bcl-2 heterodimerizes in vivo with a conserved homolog, Bax, that accelerates programmed cell death. Cell 74: 609-619, 1993.

24. Ben-Ari Z, Pappo O, Cheporko Y, Yasovich N, Offen D, Shainberg A, Leshem D, Sulkes J, Vidne BA and Hochhauser E: Bax ablation protects against hepatic ischemia/reperfusion injury in transgenic mice. Liver Transpl 13: 1181-1188, 2007.

25. Stankiewicz AR, Lachapelle G, Foo CP, Radicioni SM and Mosser DD: Hsp70 inhibits heat-induced apoptosis upstream of mitochondria by preventing Bax translocation. J Biol Chem 280 38729-38739, 2005.

26. Qu T, Huang B, Zhang L, Li L, Xu F, Huang W, Li C, Du Y and Zhang G: Identification and functional characterization of two executioner caspases in Crassostrea gigas. PLoS One 9: e89040, 2014.

27. Guan J,Li H,LvT, Chen D, Yuan Y and Qu S: Bone morphogenetic protein-7 (BMP-7) mediates ischemic preconditioning-induced ischemic tolerance via attenuating apoptosis in rat brain. Biochem Biophys Res Commun 441: 560-566, 2013.

28. D'Amelio M, Cavallucci V and Cecconi F: Neuronal caspase-3 signaling: Not only cell death. Cell Death Differ 17: 1104-1114, 2010.

29. Cory S and Adams JM: The Bcl2 family: Regulators of the cellular life-or-death switch. Nat Rev Cancer 2: 647-656, 2002.

30. Li Z and Nabel GJ: A new member of the I kappaB protein family, I kappaB epsilon, inhibits RelA (p65)-mediated NF-kappaB transcription. Mol Cell Biol 17: 6184-6190, 1997.

31. Tak PP and Firestein GS: NF-kappaB: A key role in inflammatory diseases. J Clin Invest 107: 7-11, 2001.

32. Liu Z, Wang Y, Zhao H, Zheng Q, Xiao L and Zhao M: CB2 receptor activation ameliorates the proinflammatory activity in acute lung injury induced by paraquat. Biomed Res Int 2014: 971750, 2014

33. Girotti AW: Lipid hydroperoxide generation, turnover and effector action in biological systems. J Lipid Res 39: 1529-1542, 1998.

34. Yapca OE, Borekci B, Turan MI and Gulapoglu M: The effect of agomelatine on oxidative stress induced with ischemia/reperfusion in rat ovaries. Adv Clin Exp Med 23: 715-721, 2014.

35. Lavelli V, Peri $\mathrm{C}$ and Rizzolo A: Antioxidant activity of tomato products as studied by model reactions using xanthine oxidase, myeloperoxidase and copper-induced lipid peroxidation. J Agric Food Chem 48: 1442-1448, 2000.

36. Peralta C, Fernández L, Panés J, Prats N, Sans M, Piqué JM, Gelpí E and Roselló-Catafau J: Preconditioning protects against systemic disorders associated with hepatic ischemia-reperfusion through blockade of tumor necrosis factor-induced P-selectin up-regulation in the rat. Hepatology 33: 100-113, 2001.

37. Peralta C, Hotter G, Closa D, Prats N, Xaus C, Gelpí E and Roselló-Catafau J: The protective role of adenosine in inducing nitric oxide synthesis in rat liver ischemia preconditioning is mediated by activation of adenosine A2 receptors. Hepatology 29: $126-132,1999$. 